\title{
The accuracy of absolute calibration of vibration pickups and a new calibration method using separate mountings
}

\author{
Akinori Yokota, Yasuo Tokita,* Hidemichi Komura and Katsumi Suzuki** \\ * Kobayashi Institute of Physical Research, \\ 3-20-41, Higashimotomachi, Kokubunji, Tokyo, 185 Japan \\ **RION Co., Ltd., \\ 3-20-41, Higashimotomachi, Kokubunji, Tokyo, 185 Japan
}

(Received 13!August 1987)

\begin{abstract}
The accuracy of the absolute calibration of vibration pickups is discussed. The effect of mounting of the laser interferometer and the vibrator is studied for the two conventional calibration methods, i.e. the fringe counting and disappearance methods. The result shows that it is preferable to mount an interferometer and a vibrator on separate heavy blocks independently for highly accurate calibration. The separate mountings make it possible to calibrate vibration pickups within $0.2 \%$ total error. A new calibration method using separate mountings is proposed, and effectiveness of this method is examined by comparing with these two conventional methods. This new method covers a wide frequency range including the frequency ranges of the conventional methods. In addition, phase lag from a vibrating surface to a pickup is measured by this method.
\end{abstract}

PACS number: 43. 40. Yg

\section{INTRODUCTION}

Two absolute calibration methods of vibration pickups are described in the ISO/DIS5347 as a draft international standard. One is a laser interferometric method and the other is a reciprocity method. Whichever method is used, careful consideration is required to set up the calibration system. It is important to estimate in advance the measurement error of the calibration system which is going to set up.

The measurement error of the calibration consists of systematic error and accidental error. Systematic error is almost constant for the same appliance. To realize high accuracy in calibration, it is necessary to minimize accidental error. Among various sources of accidental error, external vibration noise is one of the most important ones. In the ISO draft, it is recommended that an interferometer and a vibrator should be mounted independently on heavy isolation blocks suspended by springs whose resonance frequency is from $1 \mathrm{~Hz}$ to $2 \mathrm{~Hz}$. If the interferometer and the vibrator are mounted on a single block, the reaction of the vibrator affects the interferometer through the mounting block. The separation of the mountings is effective in order to avoid the reaction of the vibrator to the interferometer, but is affected by external vibration noise.

This paper consists of two part; 1) The error caused by the external vibration noise in the case of separate mountings is studied theoretically in relation to different calibration methods, ${ }^{1-3}$ ) e.g. the fringe counting and fringe disappearance methods. The validity of the result is examined by computer simulation. 2) Since these methods are applied to the calibration of different frequency ranges, the frequency response in a wide frequency range can not be obtained by a single method. Moreover, the mutual verification of different methods can be made by comparing the results only in narrow overlapped frequency ranges. To improve this defect, a new analytic calibration method is proposed in this paper. This method is applied to a wide frequency range including that of the fringe counting 
and disappearance methods. The phase relation between a vibration displacement of vibrator surface and the output of a vibration pickup can also be obtained.

\section{ESTIMATION OF MEASUREMENT ERROR}

The principle of the Michelson interferometer is shown in Fig. 1. When a vibrator is excited with sinusoidal motion of frequency $\omega$, the intensity $I(t)$ of laser light detected by a photodetector is expressed as follows.

$$
I(t)=K\left\{1+\cos \frac{4 \pi}{\lambda}(L+d(t))\right\},
$$

where $L=\left(l_{1}-l_{2}\right)$ is the path length difference, $d(t)=a_{0} \cos \omega t$ is the vibration displacement of vibrator surface, $\lambda$ is the wavelength of the laser light $(0.6328 \mu \mathrm{m}$ for He-Ne laser) and $K$ is a constant. In order to calibrate vibration pickups, the amplitude of displacement $a_{0}$ must be measured exactly.

There are two fundamental methods for vibration pickups using the laser interferometric method. The fringe counting method requires counting the number of maximum points of intensity of the laser light. The fringe disappearance method necessitates finding out the zero point of the filtered intensity. The former method is usually used in the frequency range between $20 \mathrm{~Hz}$ and $400 \mathrm{~Hz}$, and the latter is used in that of $200 \sim 2,000 \mathrm{~Hz}$.

\subsection{Fringe Counting Method}

The intensity, $I(t)$ in Eq. (1) becomes maximum as the surface of vibrator moves a distance of $\lambda / 2$, since $L$ is constant. During one cycle of vibration, the

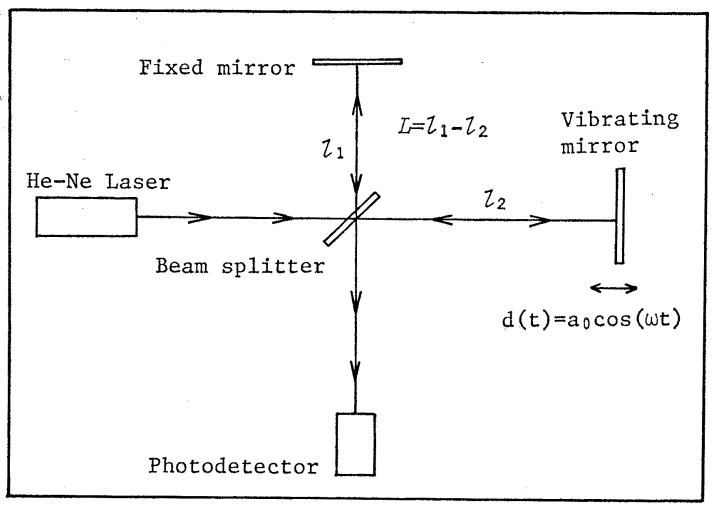

Fig. 1 Principle of the ideal interferometer. number of maxima $R$ becomes $8 a_{0} / \lambda$. $R$ equals the frequency ratio of the frequency of the fringe to the signal frequency. ${ }^{3)}$ The amplitude of displacement $a_{0}$ is obtained from the frequency ratio.

When the relative motion between the interferometer and the vibrator is excited by a vibration noise, the relative displacement is added to the displacement $d(t)$ of vibrating surface. Then, $d(t)$ in Eq. (1) becomes

$$
d(t)=a_{0} \cos \omega t+b \cos \left(\omega_{n} t+\psi\right),
$$

where $b$ is the amplitude of relative displacement between the interferometer and the vibrator, $\omega_{n}$ is the frequency of the vibration noise and $\psi$ is a phase difference between signal vibration and relative motion.

Since the frequency ratio is proportional to a distance which the vibrating surface moves, the measurement error can be calculated from a difference between the distances in the cases with and without the external vibration noise. The distance $T D$ during one cycle of the vibration noise is calculated by

$$
T D=\int_{0}^{T}|\dot{d}(t)| d t,
$$

where $T=2 \pi / \omega_{n}$, and $\dot{d}(t)$ is the time derivative of $d(t)$. In the case without the vibration noise, the distance becomes $\left(\omega / \omega_{n}\right) 4 a_{0}$ by substituting $-a_{0} \sin \omega t$ into $\dot{d}(t)$ in Eq. (3). And denoting the distance in the case with the vibration noise by $T D_{n}$, the measurement error $E_{n}$ is expressed by

$$
E_{n}=\left(\frac{T D_{n}}{\left(\omega / \omega_{n}\right) 4 a_{0}}-1\right) \times 100(\%) .
$$

Figure 2 shows the calibration error calculated by Eq. (4). $f r$ in Fig. 2 is the ratio of signal frequency to noise frequency. In the calculation, the phase

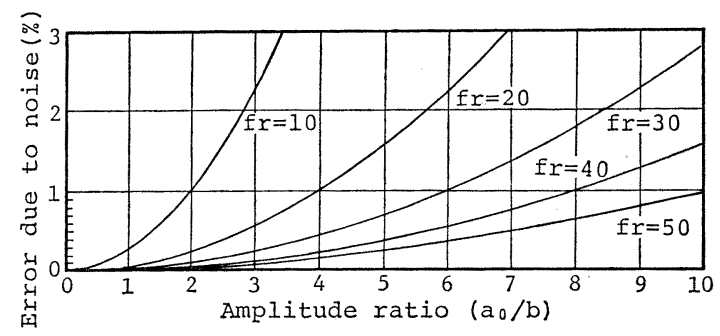

Fig. 2 Estimated calibration error caused by the external vibration noise in the case of the fringe counting method. $f r$ is the ratio of signal frequency to noise frequency. 


\section{A. YOKOTA et al.: CALIBRATION OF VIBRATION PICKUPS}

difference $\psi$ in Eq. (2) was adjusted so that the error became maximum.

A counting miss of the frequency ratio also becomes a cause of error. If the amplitude of the displacement $d(t)$ is adjusted to integral multiples of $\lambda / 2$, the counting miss can be minimized. However it is difficult to adjust the amplitude of displacement to integral multiples of $\lambda / 2$. Therefore, during one cycle of vibration signal, \pm 2 counting miss occurs in maximum. The error $E_{\mathrm{c}}$ due to counting miss is estimated by

$$
E_{\mathrm{c}}=(2 / R) \times 100(\%) .
$$

To decrease the counting miss error, it is necessary to set the amplitude large enough. In order to reduce the error less than $0.1 \%$ for $\mathrm{He}-\mathrm{Ne}$ laser light, the amplitude $a_{0}$ must be larger than $158.2 \mu \mathrm{m}$.

Two examples of the effect of the separate mountings are shown below. The calibration system is usually mounted on isolation blocks suspended by springs on the floor. The dominant frequency of the vibration noise is almost the same as the resonance frequency of the suspension. Assuming that the dominant frequency is $3 \mathrm{~Hz}$ and the signal frequency is $30 \mathrm{~Hz}$, corresponding to $f r=10$ in Fig. 2, the signal amplitude of $158.2 \mu \mathrm{m}$ permits an amplitude of vibration noise $100 \mu \mathrm{m}$ for $0.1 \%$ error. In case of $f r=50$, signal amplitude of $158.2 \mu \mathrm{m}$ permits an amplitude of vibration noise $500 \mu \mathrm{m}$ for $0.1 \%$ error. These examples show that the separate mountings are effective in order to avoid the external vibration noise.

\subsection{Fringe Disappearance Method}

The intensity of laser light given by Eq. (1) can be expanded in Fourier series and expressed as follows.

$$
\begin{aligned}
I(t)= & A+B \sum(-1)^{n} J_{2 n}(\alpha) \cos 2 n \omega t \\
& +C \sum(-1)^{2 n-1} J_{2 n-1}(\alpha) \cos (2 n-1) \omega t,(6)
\end{aligned}
$$

where $\alpha=4 \pi a_{0}$, and $A, B$ and $C$ are constant, $J_{2 n}(\alpha)$, $J_{2 n-1}(\alpha)$ are the even and odd order Bessel functions respectively. When the intensity $I(t)$ in Eq. (6) is filtered through a bandpass filter centered at an angular frequency $\omega, I(t)$ becomes

$$
I_{\mathrm{f}}(t)=-C J_{1}(\alpha) \cos \omega t .
$$

The rectified intensity $I_{\mathrm{f}}(t)_{\mathrm{rms}}$ becomes zero when $J_{1}(\alpha)=0$. The Bessel function $J_{1}(\alpha)$ is uniquely determined by the value $\alpha$, so the amplitude $a_{0}$ is obtained.

When the relative motion between the interferome- ter and the vibrator is excited by a vibration noise, the intensity of the laser light can be expressed as

$$
I(t)=\left(F_{1}+F_{2}\right) \cos \theta+\left(G_{1}+G_{2}\right) \sin \theta,
$$

where $\theta=4 L \pi / \lambda$, and $F_{1}, F_{2}, G_{1}$ and $G_{2}$ are functions expressed by Bessel functions respectively (see Appendix). And filtered intensity becomes

$$
\begin{aligned}
I_{\mathrm{f}}(t)= & J_{1}(\alpha) H_{1}(t) \sin \theta \\
& +2 J_{1}(\alpha) J_{1}(\beta) H_{2}(t) \cos \theta \\
& +\left\{\frac{1}{2}(-1)^{\left(\omega+\omega_{n}\right) / \omega_{n}} J_{0}(\alpha) J_{\omega / \omega_{n}}(\beta) \cos \theta\right. \\
& +2(-1)^{\left(\omega-2 \omega_{n}\right) / 2 \omega_{n}} J_{0}(\alpha) J_{\left(\omega-\omega_{n}\right) / \omega_{n}}(\beta) \sin \theta \\
& \left.+(-1)^{\left(\omega+\omega_{n}\right) / 2 \omega_{n}} J_{2}(\alpha) J_{\omega / \omega_{n}}(\beta) \sin \theta\right\} \cos \omega t,
\end{aligned}
$$

where $\alpha=4 \pi a_{0} / \lambda, \beta=4 \pi b / \lambda$, and $H_{1}(t)$ and $H_{2}(t)$ are beat signals being composed of $\cos \omega t, \cos \left(\omega t \pm \omega_{n}\right)$ and so on. Since Eq. (9) has higher order Bessel functions $J_{\omega / \omega n}(\beta)$ and so on, the rectified intensity $I_{\mathrm{f}}(t)_{\mathrm{rms}}=0$ is not always valid. It is possible to calibrate the pickups with high accuracy, if the higher order Bessel function is ignored. The calculated result of Bessel function of integer order $J_{n}(x)$ is almost zero for $2 x<n$, if $n$ is greater than about 50. The limit of the relative displacement between the interferometer and the vibrator can be given by

$$
B_{\max }=\omega \lambda / 8 \pi \omega_{n} .
$$

Figure 3 shows the result calculated by Eq. (10) for the resonance frequencies of $2 \mathrm{~Hz}, 3 \mathrm{~Hz}$ and $4 \mathrm{~Hz}$. If the noise amplitude is less than $B_{\max }$, Eq. (9) becomes

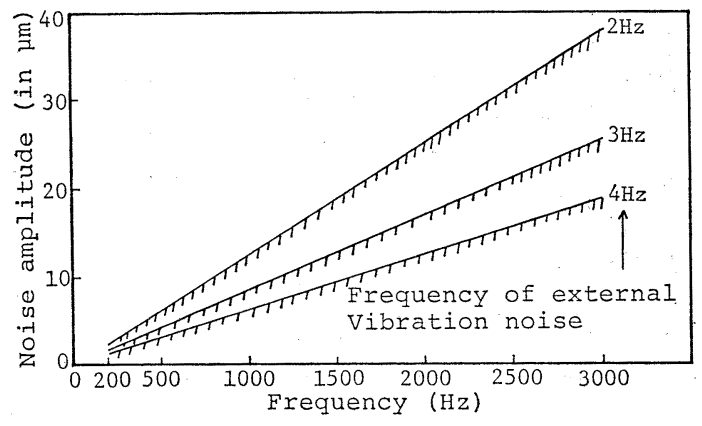

Fig. 3 Relationship between the signal frequency of vibrator and maximum noise amplitude that permits an accurate calibration (fringe counting method). 


$$
I_{\mathrm{f}}(t)=J_{1}(\alpha)\left\{H_{1}(t) \sin \theta+2 J_{1}(\beta) H_{2}(t) \cos \theta\right\} .
$$

Therefore the rectified intensity $I_{\mathrm{f}}(t)_{\mathrm{rms}}$ becomes zero at an amplitude where $J_{1}(\alpha)=0$.

Equation (11) was also examined by computer simulation using a digital filter ${ }^{4}$ shown in Fig. 4. The example of simulation is shown in Fig. 5, where (a) is AC component and (b) shows a detail view of

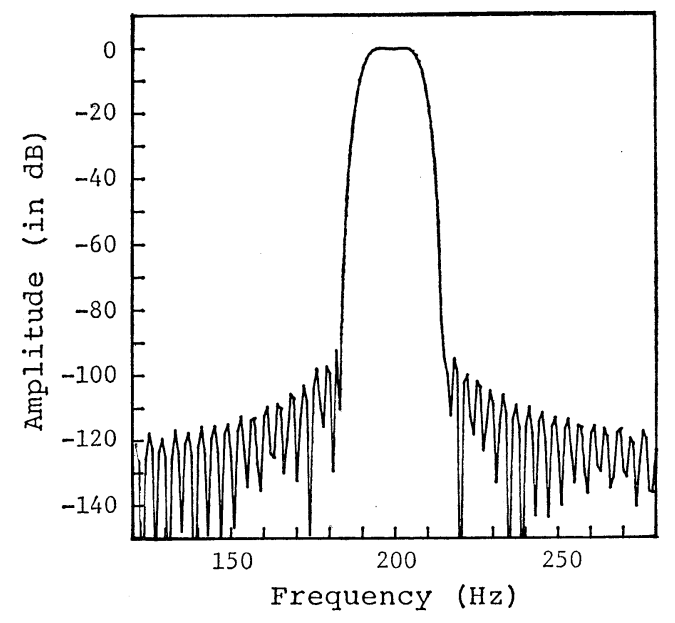

Fig. 4 Frequency response of the bandpass filter used in simulation.

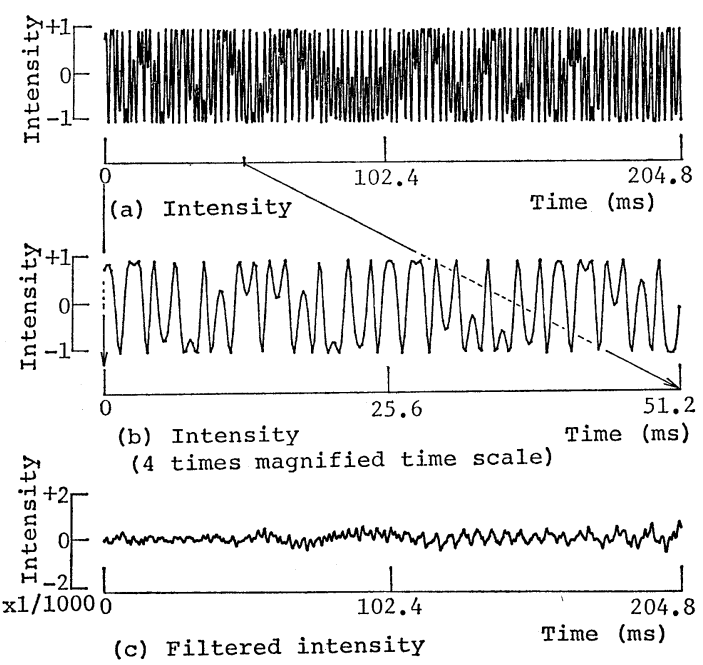

Fig. 5 An example of computer simulation of the fringe disappearance method. Frequency and amplitude of vibration signal are $200 \mathrm{~Hz}, 0.1930 \mu \mathrm{m}$ while $2 \mathrm{~Hz}, 2.5 \mu \mathrm{m}$ for noise.
AC component of the intensity. The filtered intensity (c) becomes less than $1 / 1,000$ of the original intensity. Figure 6 was obtained by changing the amplitude of noise from $2.5 \mu \mathrm{m}$ to $5 \mu \mathrm{m}$. The filtered intensity does not approach zero because of the beat signal.

Figures 7 and 8 show the relation between the rectified intensity after filtering and the amplitude of vibrator surface. The signal frequency is $200 \mathrm{~Hz}$ and the frequency of noise is $2 \mathrm{~Hz}$ in both cases, but the amplitude is $2.5 \mu \mathrm{m}$ for Fig. 7 and $5 \mu \mathrm{m}$ for Fig. 8.

The fringe disappearance method is applied to the specific amplitude corresponding to the zero point of the Bessel function. The $J_{1} / J_{3}$ method can be applied to an arbitrary amplitude of the vibrating surface. This method can be derived from the same theory as the fringe disappearance method. Using two filters, the displacement of vibrating surface is obtained from the ratio of the two harmonics. However, when the relative motion between the interferometer and the vibrator is excited, the ratio of the outputs of two filters can not be determined by Bessel functions because of beat signals. The single mounting is recommended in the case of $J_{1} / J_{3}$ method, even if the interferometer is affected by the reaction of the vibrator.

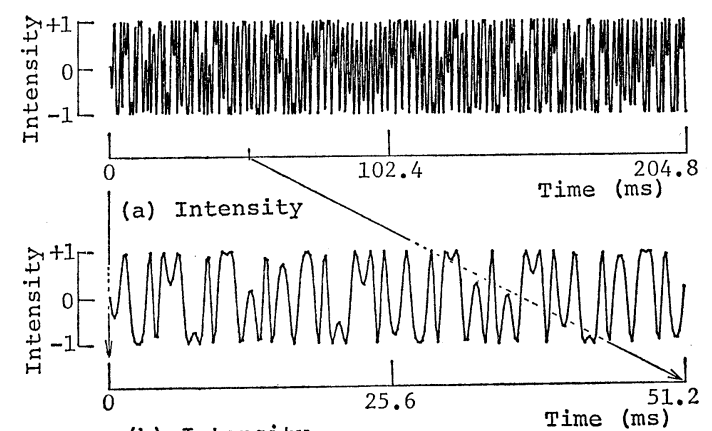

(b) Intensity Time (ms)

(4 times magnified time scale)

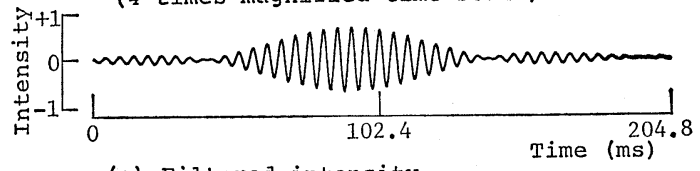

(c) Filtered intensity

Fig. 6 An example of computer simulation of the fringe disappearance method. Frequency and amplitude of vibration signal are $200 \mathrm{~Hz}, 0.1930 \mu \mathrm{m}$ while $2 \mathrm{~Hz}, 5 \mu \mathrm{m}$ for noise. 


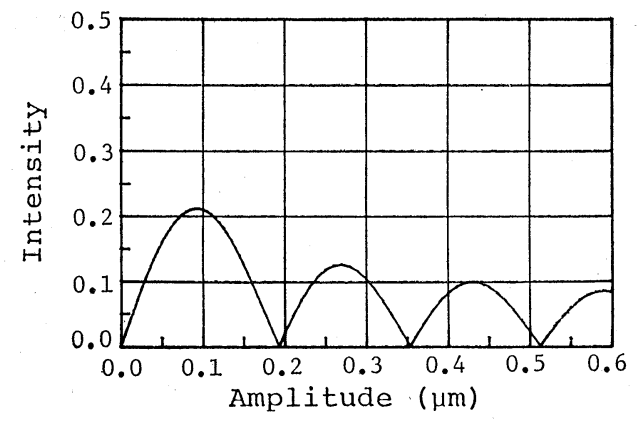

Fig. 7 Relationship between the displacement amplitude of vibrating surface and the intensity of laser light (computer simulation). Signal frequency is $200 \mathrm{~Hz}$. Frequency and amplitude of noise are $2 \mathrm{~Hz}, 2.5 \mu \mathrm{m}$.

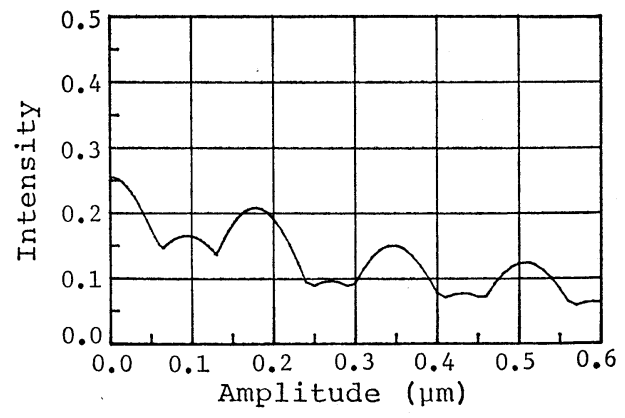

Fig. 8 Relationship between the displacement amplitude of vibrating surface and the intensity of laser light (computer simulation). Signal frequency is $200 \mathrm{~Hz}$. Frequency and amplitude of noise are $2 \mathrm{~Hz}, 5 \mu \mathrm{m}$.

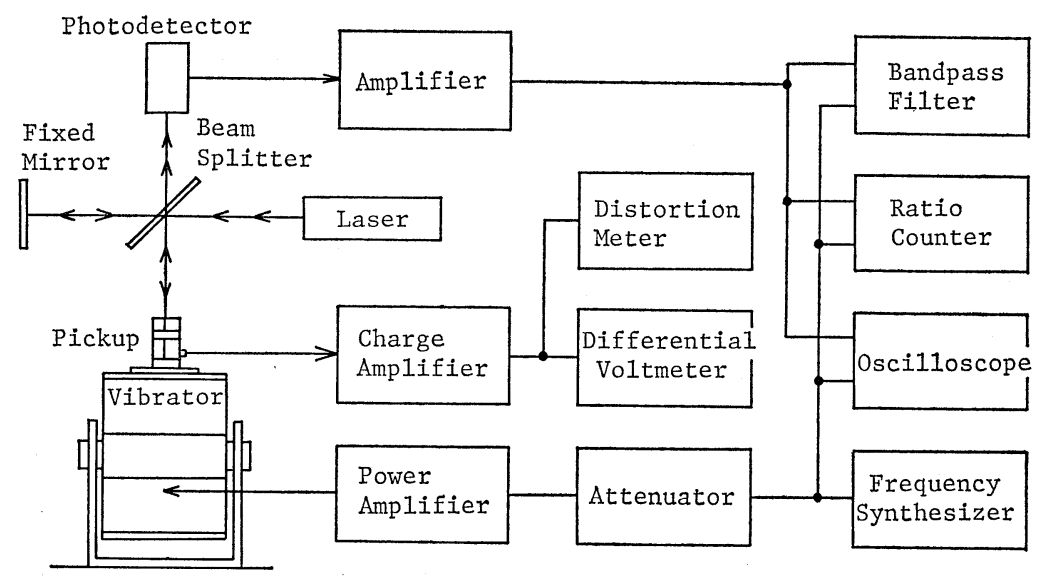

Fig. 9 Block diagram of the calibration system being used for the fringe counting and disappearance methods.

\section{EQUIPMENT FOR CALIBRATION OF VIBRATION PICKUPS}

The block diagram of the absolute calibration system is shown in Fig. 9. The photodetector, the fixed mirror, the beam splitter and the laser tube are mounted together on a $700 \mathrm{~kg}$ isolation block suspended by air springs. The resonance frequency of the suspension is $1.7 \mathrm{~Hz}$. The vibrator is mounted on a different $400 \mathrm{~kg}$ isolation block suspended by air springs. The resonance frequency is $2.5 \mathrm{~Hz}$. The illustration of mounting is shown in Fig. 10. The calibration system is placed in a semi unechoic room whose wall and ceiling are treated by fiber glass. The temperature and humidity of this room

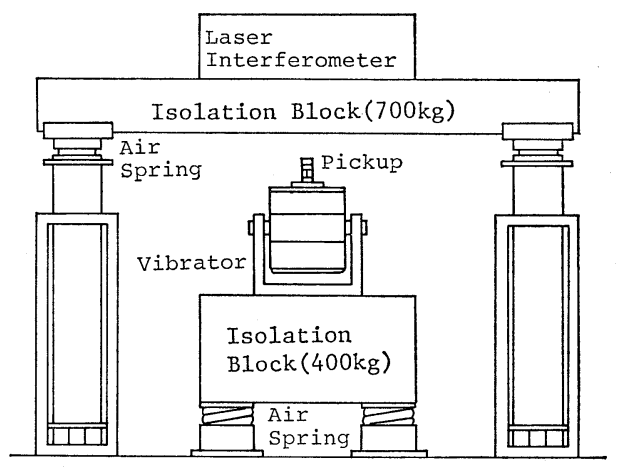

Fig. 10 Separate mountings of the interferometer and vibrator of calibration system. 
is controlled by an air conditioner to maintain the temperature between $23 \pm 3^{\circ} \mathrm{C}$ and the humidity between $50 \pm 25 \%$.

\section{THE ERROR OF CALIBRATION}

The calibrated values contain two types of error, the accidental error and the systematic error. The accidental error and the systematic error are evaluated as precision and inaccuracy in statistics.

The precision of the two calibration methods was examined using three types of standard vibration pickups, B \& K (8305), Endevco (2270) and Rion (PV-03). For every pickup, calibration was carried out four times a day and continued for 7 days. Four operators were assigned to engage in calibration once a day for every pickup. Each operator mounted the pickup on the vibrator before starting the calibration and removed it at the end of the calibration task. The pickup was affixed to the vibrator by a mounting stud with mounting torque of $2 \mathrm{Nm} .{ }^{5}$ ) The pickup had a dummy load of $20 \mathrm{~g}$ and its upper surface was polished smoothly to reflect the laser light.

The result of fringe counting method is shown in Fig. 11. The calibration was performed at frequencies of $80 \mathrm{~Hz}$ and $160 \mathrm{~Hz}$ with the acceleration of $100 \mathrm{~ms}^{-2}{ }_{\mathrm{rms}}$. The frequency and the error are expressed in percentage. The error is expressed as a relative value around the average. The result shows the precision of calibration is less than $0.11 \%$ in all cases.

Figure 12 shows the result of the fringe disappearance method. The exciting frequencies are $1 \mathrm{kHz}$ and $2 \mathrm{kHz}$. The amplitudes of the displacement of vibrating surface were adjusted to

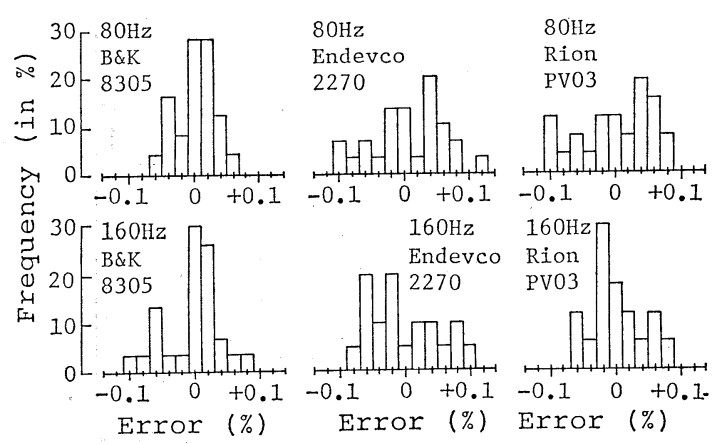

Fig. 11 Histogram showing the precision of the fringe counting method.

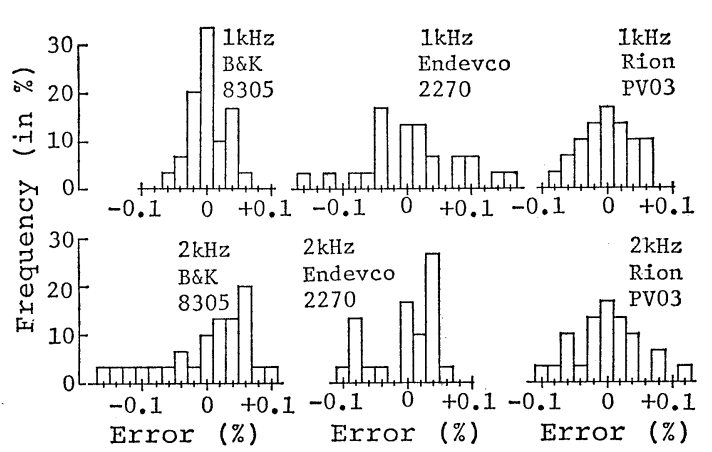

Fig. 12 Histogram showing the precision of the fringe disappearance method.

$0.1930 \mu \mathrm{m}$ at $2 \mathrm{kHz}$ and $0.6710 \mu \mathrm{m}$ at $1 \mathrm{kHz}$. They correspond to the first and fourth roots of the first order Bessel function $J_{1}(\alpha)$ in Eqs. (7) and (11). It can be seen that the precision of calibration is less than $0.13 \%$.

Inaccuracy is caused by the measuring apparatuses, especially the voltage meter, ratio counter and oscillator. The inaccuracies of the fringe counting and fringe disappearance methods were calculated according to formulas. ${ }^{6}$ ) The inaccuracy of the fringe counting method was $0.12 \%$ and that of the fringe disappearance method was $0.13 \%$.

Then total errors of the calibration are $\sqrt{0.11^{2}+0.12^{2}}=0.16 \%$ for the first method and $\sqrt{0.13^{2}+0.13^{2}}=0.18 \%$ for the second method. These results show that the measurement error caused by an external vibration noise can be reduced to less than $0.2 \%$ by separate mountings.

\section{NEW CALIBRATION METHOD (CURVE FITTING METHOD)}

Although the fringe counting method is able to calibrate vibration pickups with high accuracy, it requires an amplitude larger than $16 \mu \mathrm{m}$ of vibration displacement. On the other hand, the fringe disappearance method can calibrate accurately at rather small displacements from $0.1930 \mu \mathrm{m}$ to about $1 \mu \mathrm{m}$. The frequency ranges covered by these two methods are different. We propose a new calibration method (CFM) that covers a very wide frequency range including the above stated methods. This method is realized by using separate mountings.

\subsection{Principle}

The normalized intensity of laser light without 


\section{A. YOKOTA et al.: CALIBRATION OF VIBRATION PICKUPS}

DC component is expressed as,

$$
I(t)=\cos \left(\frac{4 \pi}{\lambda}(d(t))+\theta\right),
$$

where

$$
d(t)=a_{0} \cos \left(\omega t+\phi_{\mathrm{d}}\right)+n(t) .
$$

$a_{0} \cos \left(\omega t+\phi_{\mathrm{d}}\right)$ is the displacement of the vibrator surface, and $n(t)$ is the relative displacement being excited by an external vibration noise between the interferometer and the vibrator. Since the frequency ratio of signal to vibration noise is large enough, $n(t)$ can be approximated by a linear function of time $t$ during one or two signal periods. The inverse function of $I(t)$ is given by

$$
\cos ^{-1} I(t)=\frac{4 \pi}{\lambda}\left(a_{0} \cos \left(\omega t+\phi_{\mathrm{d}}\right)+a_{n} t\right)+\theta .
$$

Now, putting $Y(t)=\cos ^{-1} I(t), X_{1}(t)=\cos \omega t, X_{2}(t)=$ $\sin \omega t, \quad X_{3}(t)=t, \quad A_{0}=-4 \pi a_{0} \cos \phi_{\mathrm{a}} / \lambda, \quad B_{0}=-4 \pi a_{0}$ $\times \sin \phi_{\mathrm{d}} / \lambda$ and $C_{0}=4 \pi a_{n}$, Eq. (14) becomes

$$
Y(t)=A_{0} X_{1}(t)+B_{0} X_{2}(t)+C_{0} X_{3}(t)+\theta .
$$

The coefficients $A_{0}, B_{0}$, and $C_{0}$ are solved by multiple regression analysis. Since the laser wavelength $\lambda$ is known and the exciting frequency $\omega$ and time $t$ can be measured exactly, the amplitude and phase of vibrating displacement of the vibrator surface are solved by

$$
\begin{aligned}
& \phi_{\mathrm{d}}=\tan ^{-1}\left(B_{0} / A_{0}\right) \\
& a_{0}=A_{0} \lambda / 4 \pi \cos \phi_{\mathrm{d}} .
\end{aligned}
$$

\subsection{Computational Procedure}

Figure 13 shows the block diagram of the measur- ing instruments used in the newly developed method. The intensity $I(t)$ of the laser light and the acceleration signal of pickup output are simultaneously sampled and stored in a transient memory. The size of memory is $1 \mathrm{k}$ words for each channel. The data stored in the transient memory are transferred to a personal computer for the computation. Then, the data are transformed to $Y_{i}, i=1,2,3, \cdots$ using the inverse trigonometric function. The value of $Y_{i}$ varies only in the range from 0 to $\pi$. To obtain a smooth waveform, the sequence $Y_{i}$ must be unwrapped by adding or subtracting $n \pi$. Since the waveform of $d(t)$ in Eq. (13) is simple, unwrapping is simply processed as follows;

First, the 11-th sample $Y_{11}$ is estimated from the first 10 data samples using the regression analysis. Comparing the measured 11-th sample value with the estimated one, an integral multiple of $\pi$ is added to or subtracted from the 11-th sample so as to minimize the difference. The 12-th sample $Y_{12}$ is processed from 11 samples in the same way as the procedure to correct the 11-th sample, except using corrected $Y_{11}$. The same procedure is repeated sequentially till the 210-th sample. The length of 200 samples is almost equal to the quarter of the wavelength. From the 211-th sample to 1,000-th sample, the unwrapping procedure is carried out using only the preceding corrected 200 samples.

After the unwrapping procedure, the coefficients of Eq. (15) are evaluated from the multiple regression analysis. Then the amplitude $a_{0}$ and the phase $\phi_{\mathrm{d}}$ of the displacement of reference surface are computed using Eqs. (16) and (17). An example

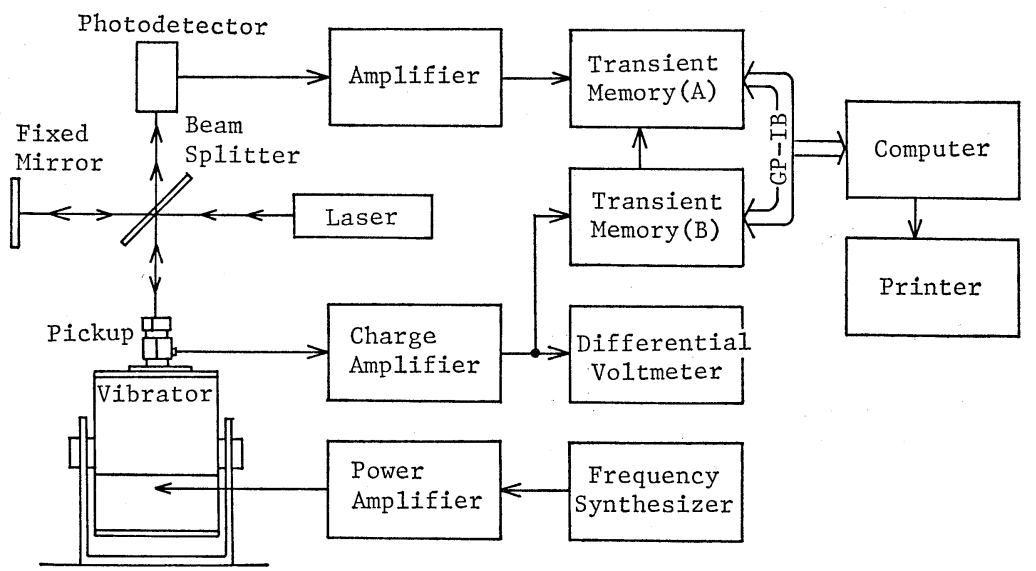

Fig. 13 Block diagram of the calibration system being used for the curve fitting method. 
of this procedure is shown in Fig. 14. Figure 14(a) shows the initial intensity $I(t),(b)$ is the computed inverse trigonometric function $Y(t)$, and (c) is the result of unwrapping, that is, the estimated waveform of displacement of the vibrating surface.

The waveform of acceleration, which is observed through a charge amplifier, is able to be expressed as follows,

$$
p(t)=p_{0} \cos \left(\omega t+\phi_{\mathrm{p}}\right) .
$$

The phase $\phi_{\mathrm{p}}$ is obtained from a regression analysis of $p(t)$ in a similar way as the waveform of displacement. The difference between $\phi_{\mathrm{d}}+\pi$ and $\phi_{\mathrm{p}}$ gives the phase lag from the vibrator surface to the pickup.

Figure 15 shows the sensitivity and phase curves of a standard pickup (Rion PV-03) estimated by
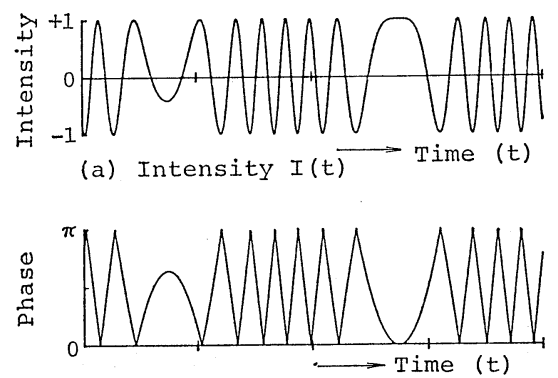

(b). Inverse function of $I(t)$

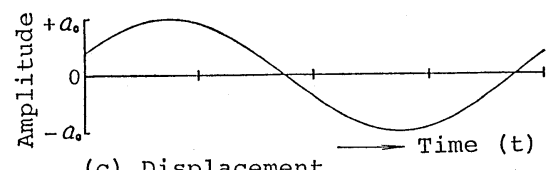

(c) Displacement

Fig. 14 Waveforms of the intensity, the inverse function of intensity and the displacement.

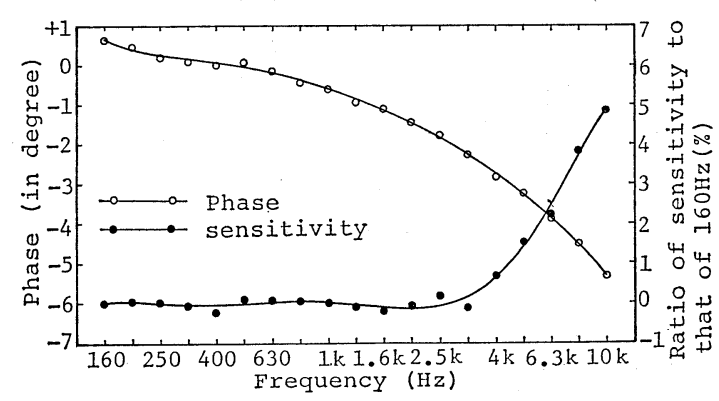

Fig. 15 Sensitivity and phase characteristics measured by the curve fitting method. Standard pickup is Rion PV-03.
CFM. The sensitivity of the pickup was obtained from the measurement of the vibration displacement on the surface of a dummy load. The curve of the sensitivity is normalized by the value at $160 \mathrm{~Hz}$. The phase is positive below $400 \mathrm{~Hz}$, because the phase $\phi_{\mathrm{p}}$ includes the phase characteristics of the charge amplifier. In the frequency range between $160 \mathrm{~Hz}$ and $315 \mathrm{~Hz}$, the amplitude of the displacement of the surface was controlled to be about 16 $\mu \mathrm{m}$, and between $3.15 \mathrm{kHz}$ and $10 \mathrm{kHz}$, it was 0.25 $\mu \mathrm{m}$ to $0.15 \mu \mathrm{m}$. For $400 \mathrm{~Hz} \sim 2.5 \mathrm{kHz}$, the amplitude of acceleration was controlled to be about $450 \mathrm{~ms}^{-2}{ }_{\mathrm{rms}}$. An electrodynamic vibrator was used in the frequency range below $2 \mathrm{kHz}$. A piezoelectric vibrator ${ }^{\text {?) }}$ was used above $2.5 \mathrm{kHz}$.

\section{COMPARISON OF THE ACCURACY OF CFM WITH THE CONVENTIONAL TWO METHODS}

As is already shown in Chap. 4, vibration pickups can be calibrated accurately by the fringe counting and fringe disappearance methods, but the effective frequency ranges of these methods are different. The CFM can cover a wide frequency range including those of two methods mentioned above. The accuracy of the CFM is compared with those of the two methods.

First, CFM and fringe disappearance method were compared with each other at $1 \mathrm{kHz}$ for four amplitudes of the displacement corresponding to the 1-st, 2-nd, 3-rd and 4-th minimal points of the first order Bessel function. In order to execute the calibration under the same condition, the pickup was affixed to the vibrator by torque of $2 \mathrm{Nm}$ at the beginning of measurement and was kept constant during the series of measurement. The results are shown in Table 1. The difference in sensitivities calibrated by these two methods is less than $0.07 \%$.

Secondly, the same pickup was calibrated by the CFM and fringe counting method at $400 \mathrm{~Hz}$ for 6 different amplitudes of the displacement. They correspond to the frequency ratio of $10,20,50,100$, 200 and 500. Table 2 shows the sensitivity measured by these two methods. The differences between the sensitivity at the amplitude of $16 \mu \mathrm{m}$ of CFM and others are shown in percentage. It can be seen that the difference in the sensitivity between these two methods is comparatively large at amplitudes below $8 \mu \mathrm{m}$, while it is small above $16 \mu \mathrm{m}$. This result is reasonable because the fringe counting 


\section{A. YOKOTA et al.: CALIBRATION OF VIBRATION PICKUPS}

Table 1 Comparison between the fringe disappearance method and the curve fitting method. Standard pickup Rion PV-03 was calibrated at $1 \mathrm{kHz}$.

\begin{tabular}{lcccc}
\hline & $\begin{array}{c}\text { Displace- } \\
\text { ment } \\
(\mu \mathrm{m})\end{array}$ & $\begin{array}{c}\text { Disappear- } \\
\text { ance } \\
(\mathrm{mV} / \mathrm{G})(A)\end{array}$ & $\begin{array}{c}\text { Curve } \\
\text { fitting } \\
(\mathrm{mV} / \mathrm{G})(B)\end{array}$ & \begin{tabular}{c}
$(A-B) \cdot 100$ \\
\cline { 5 - 5 }$(\%)$
\end{tabular} \\
\hline 1 & 0.1930 & 42.15 & 42.17 & -0.05 \\
2 & 0.3533 & 42.13 & 42.12 & 0.02 \\
3 & 0.5123 & 42.14 & 42.13 & 0.02 \\
4 & 0.6710 & 42.15 & 42.12 & 0.07 \\
\hline
\end{tabular}

Table 2 Comparison between the fringe counting method and the curve fitting method. Standard pickup Rion PV-03 was calibrated at $400 \mathrm{~Hz}$.

\begin{tabular}{cccccc}
\hline $\begin{array}{c}\text { Displace- } \\
\text { ment } \\
(\mu \mathrm{m})\end{array}$ & \multicolumn{2}{c}{ Fringe counting } & & \multicolumn{2}{c}{ Curve fitting } \\
\cline { 2 - 3 } \cline { 5 - 6 } \cline { 5 - 6 } & $\mathrm{mV} / \mathrm{G}$ & $\%$ & & $\mathrm{mV} / \mathrm{G}$ & $\%$ \\
\hline 40 & 42.15 & +0.05 & & - & - \\
16 & 42.16 & +0.07 & & 42.13 & Base \\
8 & 42.20 & +0.17 & & 42.13 & 0 \\
4 & 42.27 & +0.38 & & 42.13 & 0 \\
1.6 & 42.54 & +0.95 & & 42.12 & -0.02 \\
0.8 & 42.97 & +1.99 & & 42.14 & +0.02 \\
\hline
\end{tabular}

method is usually used at large amplitude of displacement for highly accurate calibration.

\section{CONCLUDING REMARKS}

The influence of an external vibration noise was studied on the fringe counting and fringe disappearance methods. The result shows it is better to mount the interferometer and the vibrator separately in order to isolate the influence of the vibration noise, and that the separate mountings make it possible to calibrate vibration pickups very accurately within a total error of $0.2 \%$.

The new CFM method was proposed to cover a wide frequency range which includes frequency ranges of the fringe counting and disappearance methods. The accuracy of the CFM was verified in comparison with these two methods. The differences in the sensitivities were less than $0.1 \%$. It shows that CFM is available to supplement the frequency range in which vibration pickups can not be calibrated with high accuracy by the two methods.

\section{ACKNOWLEDGEMENTS}

The authors wish to thank to Mr. K. Taya, M. Araki and M. Hata for their serious discussion and measurement. Thanks also to Mr. I. Yamada for his helpful advice and for his careful reading. Finally, the authors are also grateful to Professor J. Igarashi for his helpful advice and encouragement.

\section{REFERENCES}

1) V. A. Schmidt, E. R. Smith, and E. Jones, "Optical calibration of vibration pickups at small amplitude," J. Acoust. Soc. Am. 33, 748-751 (1961).

2) H. A. Deferrari and F. A. Andrews, "Vibration displacement and mode-shape measurement by a laser interferometer," J. Acoust. Soc. Am. 42, 982990 (1967).

3) ISO/DIS5347, "Methods of calibration of vibration and shock pickups" (1984).

4) L. R. Rabiner and R. W. Schafer, Digital Processing of Speech Signal (Prentice-Hall, Englewood Cliffs, 1987).

5) ISO/DIS5348, "Mechanical mounting of accelerometer" (1980).

6) ISO/DP5347/DAD 1, "Primary vibration calibration by laser interferometer" (1981).

7) E. Jones, W. B. Yelon, and S. Edelman, "Piezoelectric shaker for wide-frequency calibration of vibration pickups," J. Acoust. Soc. Am. 45, 15561559 (1969).

\section{APPENDIX}

To prove Eq. (8) is not difficult, but it requires many pages so the result is only shown. $F_{1}, F_{2}, G_{1}$ and $G_{2}$ in Eq. (8) are as follows.

$$
\begin{aligned}
F_{1}= & J_{0}(\alpha) J_{0}(\beta)+J_{0}(\beta) \sum(-1)^{n} J_{2 n}(\alpha) \cos (2 n \omega t) \\
& +J_{0}(\alpha) \sum(-1)^{n} J_{2 n}(\beta) \cos \left(2 n \omega_{n} t\right) \cos (2 n \psi) \\
& +J_{0}(\alpha) \sum(-1)^{n} J_{2 n}(\beta) \sin \left(2 n \omega_{n} t\right) \sin (2 n \psi) \\
& +(1 / 2) \sum \sum(-1)^{n+k} J_{2 n}(\alpha) J_{2 k}(\beta) \cos (2 k \psi) \\
& +\left\{\cos \left(2 n \omega+2 k \omega_{n}\right) t+\cos \left(2 n \omega-2 k \omega_{n}\right) t\right\} \\
& +(1 / 2) \sum \sum(-1)^{n+k} J_{2 n}(\alpha) J_{2 k}(\beta) \sin (2 k \psi) \\
& +\left\{\sin \left(2 n \omega+2 k \omega_{n}\right) t-\sin \left(2 n \omega-2 k \omega_{n}\right) t\right\}, \\
F_{2}= & 2 \sum \sum(-1)^{n+k-2} J_{2 n-1}(\alpha) J_{2 k-1}(\beta)[\cos \{(2 n-1) \omega t \\
& \left.+(2 k-1) \omega_{n} t\right\} \\
& \left.+\cos \left\{(2 n-1) \omega t-(2 k-1) \omega_{n} t\right\}\right] \cos \{(2 k-1) \psi\} \\
& +2 \sum \sum(-1)^{n+k-2} J_{2 n-1}(\alpha) J_{2 k-1}(\beta)[\sin \{(2 n-1) \omega t \\
& \left.+(2 k-1) \omega_{n} t\right\} \\
& \left.-\sin \left\{(2 n-1) \omega t-(2 k-1) \omega_{n} t\right\}\right] \sin \{(2 k-1) \psi\}, \\
G_{1}= & 2 J_{0}(\beta) \sum(-1)^{n-1} J_{2 n-1}(\alpha) \cos (2 n-1) \omega t
\end{aligned}
$$




$$
\begin{aligned}
& +\sum \sum(-1)^{n+k-1} J_{2 n-1}(\alpha) J_{2 k}(\beta)[\cos \{(2 n-1) \omega t \\
& \left.\left.+2 k \omega_{n} t\right\}+\cos \left\{(2 n-1) \omega t-2 k \omega_{n} t\right\}\right] \cos (2 k \psi) \\
& +\sum \sum(-1)^{n+k-1} J_{2 n-1}(\alpha) J_{2 k}(\beta)[\sin \{(2 n-1) \omega t \\
& \left.\left.+2 k \omega_{n} t\right\}-\sin \left\{(2 n-1) \omega t-2 k \omega_{n} t\right\}\right] \sin (2 k \psi),
\end{aligned}
$$
and

$G_{2}=2 J_{0}(\alpha) \sum(-1)^{n} J_{2 n-1}(\beta) \cos \left(2 n \omega_{n} t\right) \cos (2 n \psi)$
$+2 J_{0}(\alpha) \sum(-1)^{n} J_{2 n-1}(\beta) \sin \left(2 n \omega_{n} t\right) \sin (2 n \psi)$

$+\sum \sum(-1)^{n+k-1} J_{2 n}(\alpha) J_{2 k-1}(\beta)[\cos \{2 n \omega t$

$\left.\left.+(2 k-1) \omega_{n} t\right\}+\cos \left\{2 n \omega t-(2 k-1) \omega_{n} t\right\}\right]$

$\cdot \cos (2 k-1) \psi+\sum \sum(-1)^{n+k-1} J_{2 n}(\alpha) J_{2 k-1}(\beta)$

- $\left[\sin \left\{2 n \omega t+(2 k-1) \omega_{n} t\right\}\right.$

$\left.-\sin \left\{2 n \omega t-(2 k-1) \omega_{n} t\right\}\right] \sin (2 k-1) \psi$. 